\title{
A Table of the First Factor for Prime Cyclotomic Fields
}

\author{
By Morris Newman
}

\begin{abstract}
The first factor of the prime cyclotomic fields for all primes $<200$ is computed by means of a determinantal formula, correcting some errors in tables of Kummer.
\end{abstract}

Let $p=2 m+1$ be an odd prime. Let $\zeta$ be a primitive $p$ th root of unity, and let $R$ be the field of rationals. It is well known that if $h$ is the class number of the cyclotomic field $R(\zeta)$, and $h_{0}$ the class number of the totally real subfield $R(\zeta+1 / \zeta)$, then $h$ is divisible by $h_{0}$. The quotient $h / h_{0}$ is denoted by $h^{*}$, and is known as the first factor of $h$. A complete discussion of these matters is to be found in the beautiful book [1] by Borevič and Šafarevič, where a table (uncredited) of $h^{*}$ is given for all odd primes $p<100$. Presumably, this table is due to Kummer, who computed $h^{*}$ for all odd primes $\leqq 163$ (see [2] and [3]). The numbers $h^{*}$ are quite difficult to compute, and it is of some interest to verify and to extend the above-mentioned tables. The importance of $h^{*}$ stems from the fact that the prime $p$ is irregular if and only if $p$ divides $h^{*}$.

It turns out that Kummer's tables are not error-free: the values of $h^{*}$ corresponding to $p=103,139$, and 163 are incorrect. The fact that $p=103$ is irregular, and was correctly identified to be so by Kummer, is explicable by his method of computation.

Let $g$ be a primitive root modulo $p$. Define

$$
g_{n}=g^{n}-p\left[g^{n} / p\right], \quad n=0,1,2, \cdots .
$$

Let $\theta$ be a primitive $(p-1)$ st root of unity. Then the first factor $h^{*}$ is given by the formula

$$
h^{*}=\frac{1}{(2 p)^{m-1}} F(\theta) F\left(\theta^{3}\right) \cdots F\left(\theta^{p-2}\right) \text {, }
$$

where

$$
F(x)=\sum_{n=0}^{p-2} g_{n} x^{n}
$$

Kummer expresses $h^{*}$ as the product of norms

$$
h^{*}=\frac{1}{(2 p)^{m-1}} \prod_{d \mid m ; d \text { odd }} N\left\{F\left(\theta^{d}\right)\right\},
$$

and computes each rational integral factor $N\left\{F\left(\theta^{d}\right)\right\}$ separately. Thus an error in the computation of $h^{*}$ can occur without violating the divisibility (or nondivisibility)

Received June 5, 1969, revised August 7, 1969.

AMS Subject Classifications. Primary 1002; Secondary 1066.

Key Words and Phrases. Cyclotomic fields, class numbers, tables, factorizations. 
of $h^{*}$ by $p$, if the error occurs in the proper place. Notice that the formula above indicates that if $m$ has many odd divisors, then $h^{*}$ can be expected to have many factors.

The number $h^{*}$ can also be expressed as a determinant. The companion matrix of the $m \times m$ diagonal matrix diag $\left(\theta, \theta^{3}, \cdots, \theta^{p-2}\right)$ is the generalized permutation matrix

$$
Q=\left[\begin{array}{rrrrr}
0 & 1 & 0 & \cdots & 0 \\
0 & 0 & 1 & \cdots & 0 \\
& . & \cdot & \cdot & \\
0 & 0 & 0 & \cdots & 1 \\
-1 & 0 & 0 & \cdots & 0
\end{array}\right]
$$

which of course is nonderogatory and satisfies $Q^{m}+I=0$. Therefore, the determinant of the matrix $F(Q)$ is just $(2 p)^{m-1} h^{*}$, and this leads (after some elementary rearrangements) to the formula

$$
\begin{gathered}
(2 p)^{m-1} h^{*}=|\operatorname{det}(A)|, \\
A=\left(g_{m+i+j}-g_{i+j}\right), \quad 0 \leqq i, \quad j \leqq m-1,
\end{gathered}
$$

given as problem 5 on p. 367 of [1].

This formula is somewhat unsatisfactory however, because of the occurrence of the factor $(2 p)^{m-1}$. This can be remedied very simply by taking into account the relationships

$$
g_{i+j} \equiv g_{i} g_{j} \bmod p, \quad g_{i+m}+g_{i}=p .
$$

Subtract row 0 of $A$ from every other row. A factor 2 now occurs throughout the last $m-1$ rows. Remove this factor of $2^{m-1}$, and then subtract $g_{j}$ times column 0 from column $j, 1 \leqq j \leqq m-1$. A factor $p$ now occurs throughout the last $m-1$ columns. Remove this factor of $p^{m-1}$. The result is that

$$
h^{*}=|\operatorname{det}(B)|, \quad B=\left(b_{i j}\right), \quad 0 \leqq i, j \leqq m-1,
$$

where

$$
\begin{array}{ll}
b_{00}=p-2, & \\
b_{0 j}=1-g_{j}, & 1 \leqq j \leqq m-1, \\
b_{i 0}=1-g_{i}, & 1 \leqq i \leqq m-1, \\
b_{i j}=\left(g_{i} g_{j}-g_{i+j}\right) / p, & 1 \leqq i, j \leqq m-1 .
\end{array}
$$

The computation of $\operatorname{det}(B)$ for all odd primes $<200$ was carried out as a test of a program of the author's which finds the exact solution of a system of linear equations with whole number coefficients, and which also produces the determinant of the system. Storage requirements limit the program to systems of 100 equations or less, which is sufficient to accommodate the above mentioned primes. The program incorporates a check by direct multiplication which guarantees the accuracy 
of the results obtained. In addition, it is known that the irregular primes under 200 are $p=37,59,67,101,103,131,149,157$; and in each of these instances $h^{*}$ is divisible by $p$, furnishing an additional check on the computation. For $p=157, h^{*}$ is divisible by $p^{2}$, as was already noticed by Kummer [3]. This suggests the conjecture: There are infinitely many primes $p$ such that $h^{*}$ is divisible by $p^{2}$, or indeed

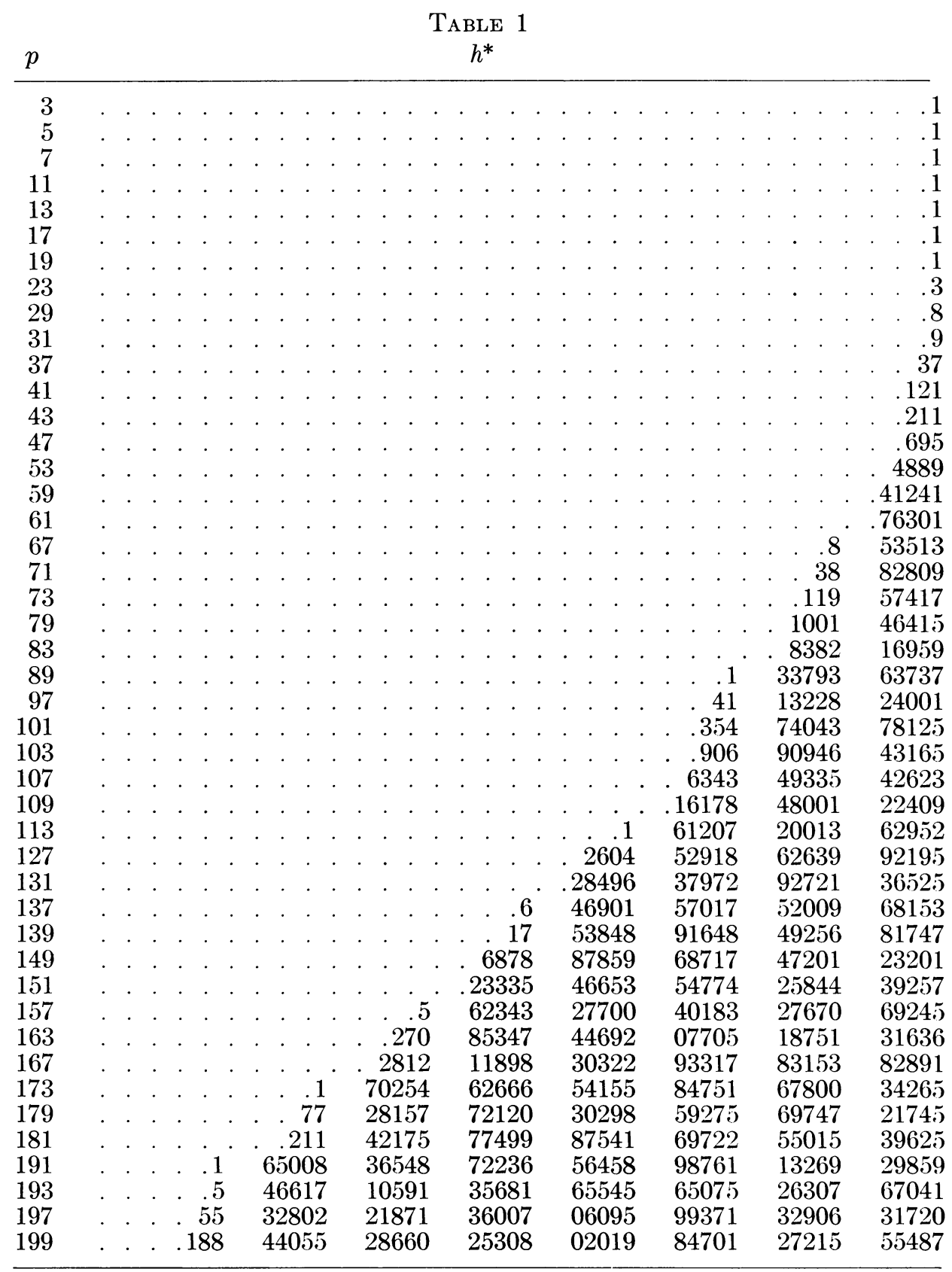


TABLE 2

p Factorization of $h^{*}$

\begin{tabular}{|c|c|}
\hline & \\
\hline 3 & - \\
\hline 5 & - \\
\hline 7 & - \\
\hline 11 & - \\
\hline 13 & - \\
\hline 17 & - \\
\hline 19 & - \\
\hline 23 & 3 \\
\hline 29 & $2 \cdot 2 \cdot 2$ \\
\hline 31 & $3 \cdot 3$ \\
\hline 37 & 37 \\
\hline 41 & $11 \cdot 11$ \\
\hline 43 & 211 \\
\hline 47 & $5 \cdot 139$ \\
\hline 53 & 4889 \\
\hline 59 & $3 \cdot 59 \cdot 233$ \\
\hline 61 & $41 \cdot 1861$ \\
\hline 67 & $67 \cdot 12739$ \\
\hline 71 & $7 \cdot 7 \cdot 79241$ \\
\hline 73 & $89 \cdot 134353$ \\
\hline 79 & $5 \cdot 53 \cdot 377911$ \\
\hline 83 & $3 \cdot 279405653$ \\
\hline 89 & $113 \cdot 118401449$ \\
\hline 97 & $577 \cdot 3457 \cdot 206209$ \\
\hline 101 & $5 \cdot 5 \cdot 5 \cdot 5 \cdot 5 \cdot 101 \cdot 601 \cdot 18701$ \\
\hline 103 & $5 \cdot 103 \cdot 1021 \cdot 17247691$ \\
\hline 107 & $3 \cdot 743 \cdot 9859 \cdot 2886593$ \\
\hline 109 & $17 \cdot 1009 \cdot 9431866153$ \\
\hline 113 & $2 \cdot 2 \cdot 2 \cdot 17 \cdot 11853470598257$ \\
\hline 127 & $5 \cdot 13 \cdot 43 \cdot 547 \cdot 883 \cdot 3079 \cdot 626599$ \\
\hline 131 & $3 \cdot 3 \cdot 3 \cdot 5 \cdot 5 \cdot 53 \cdot 131 \cdot 1301 \cdot 4673706701$ \\
\hline 137 & $17 \cdot 17 \cdot 47737 \cdot 46890540621121$ \\
\hline 139 & $3 \cdot 3 \cdot 47 \cdot 47 \cdot 277 \cdot 277 \cdot 967 \cdot 1188961909$ \\
\hline 149 & $3 \cdot 3 \cdot 149 \cdot 512966338320040805461$ \\
\hline 151 & $7 \cdot 11 \cdot 11 \cdot 281 \cdot 25951 \cdot 1207501 \cdot 312885301$ \\
\hline 157 & $5 \cdot 13 \cdot 13 \cdot 157 \cdot 157 \cdot 1093 \cdot 1873 \cdot 418861 \cdot 3148601$ \\
\hline 163 & $2 \cdot 2 \cdot 181 \cdot 23167 \cdot 365473 \cdot 441845817162679$ \\
\hline 167 & $11 \cdot 499 \cdot 5123189985484229035947419$ \\
\hline 173 & $5 \cdot 20297 \cdot 231169 \cdot 72571729362851870621$ \\
\hline 179 & $5 \cdot 1069 \cdot 14458667392334948286764635121$ \\
\hline 181 & $5 \cdot 5 \cdot 5 \cdot 37 \cdot 41 \cdot 61 \cdot 1321 \cdot 2521 \cdot 5488435782589277701$ \\
\hline 191 & $11 \cdot 13 \cdot 51263 \cdot 612771091 \cdot 36733950669733713761$ \\
\hline 193 & $6529 \cdot 15361 \cdot 29761 \cdot 91969 \cdot 10369729 \cdot 192026280449$ \\
\hline 197 & $2 \cdot 2 \cdot 2 \cdot 5 \cdot 1877 \cdot 7841 \cdot 9398302684870866656225611549$ \\
\hline 199 & $3 \cdot 3 \cdot 3 \cdot 3 \cdot 19 \cdot 727 \cdot 25645093 \cdot 207293548177 \cdot 3168190412839$ \\
\hline
\end{tabular}

by any given power of $p$. The conjecture is true for the first power, since it is known that there are infinitely many irregular primes. Vandiver has proved that $h^{*}$ is divisible by $p^{2}$ if and only if there are integers $a, b$ such that $1 \leqq a<b \leqq m-1$, and the numerators of the Bernoulli numbers $B_{2 a}, B_{2 b}$, are each divisible by $p$. 
Table 1 gives the values of $p$ and $h^{*}$. Table 2 gives the factorization of $h^{*}$, and was computed by D. H. Lehmer. The author had determined all prime factors $<10^{5}$ of $h^{*}$, but was unable to produce all factors, because of lack of machine time. Professor Lehmer kindly remedied this situation.

There are some interesting points in the tables. Thus for $p$ under $200, h^{*}$ is even only for $p=29,113,163$, and 197; and in all of these cases $h^{*}$ is divisible by 4 . There is thus a distinct possibility that $h^{*}$ is never singly even. Also $h^{*}$ is composite for all primes $p$ such that $59 \leqq p \leqq 199$, prompting the somewhat dubious conjecture that $h^{*}$ is always composite except for finitely many $p$. Also $h^{*}$ is square-free in 29 of the 45 cases given; and $29 / 45=.64444 \cdots$ does not compare too badly with $6 / \pi^{2}=.60792 \cdots$.

The computation of Table 1 was carried out on the computer of the National Bureau of Standards, and required approximately 30 minutes of machine time.

National Bureau of Standards

Washington, D. C. 20234

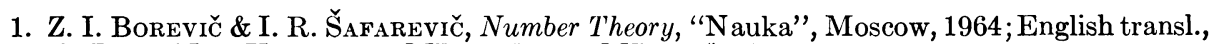
Academic Press, New York, 1966. MR 30 \#1080; MR 33 \#4001.

2. E. KuMMER, "Über die Klassenanzahl der aus $n$ ten Einheitswurzeln gebildeten complexen Zahlen," Monatsh. Preuss. Akad. Wiss. Berlin, 1861, pp. 1051-1053.

3. E. Kummer, "Über diejenigen Primzahlen $\lambda$, für welche die Klassenzahl der aus $\lambda$ ten Einheitswurzeln gebildeten complexen Zahlen durch $\lambda$ theilbar ist," Monatsh. Preuss. Akad. Wiss. Berlin, 1874, pp. 239-248. 